\title{
HAADF and EELS Analysis of Ti Deposited on Si Substrate Prepared by FIB
}

\author{
A. Medina-Flores*, L. Béjar-Gómez**, J.C. Rubio-Ávalos*, J.L. Bernal**, \\ *UMSNH. Ciudad Universitaria, Morelia, Michoacán. 58000. México. \\ **Universidad Politécnica del Valle de México. Avenida Mexiquense s/n. C.P. 54910. Tultitlán \\ Estado de México.
}

A thin slice of a transversal section of the titanium aggregates deposited on a silicon substrate was produced by Focus Ion Beam (FIB). TEM specimens were prepared, using "runscript" FEI software. A thin slice of a transversal section was cut automatically from both sides of the chosen area using an ion beam current of $5 \mathrm{nA}$. The remaining material was then automatically thinned using low currents (from $3 \mathrm{nA}$ to $300 \mathrm{pA}$ ) down to a thickness of about $300 \mathrm{~nm}$. For the last milling step, the operator cleaned a selected area down to TEM electron transparency, which is less than $100 \mathrm{~nm}$. The sample was placed at $45^{\circ}$ viewing angle and cut free by the ion beam.

The produced lamella was finally lifted out of the sample ex-situ and deposited on the carbon foil of a copper grid, ready for TEM observation. HAADF and EELS techniques were carried out to obtain the corresponding contrast of the sample, and the element mapping for each zone and the interface between them. Figure 1 shows an image of the sample obtained by HAADF technique; it is possible to observe several areas with different contrast indicating the presence of several elements which present a well-defined interface between them, this contrast is produced by the different interactions produced by the backscattered electrons which interactions whit the sample. To obtain the profile elements across the sample and observe the interface between different elements it was necessary to use the HAADF+EDS technique. On the figure 1 was marked a red line across the sample and the figure 2 shows a line scan profile along of the line. Figure $2 \mathrm{a}$ shows the element profile of $\mathrm{Ga}$ and $\mathrm{Si}$ elements and the figure $2 \mathrm{~b}$ shows the $\mathrm{Pt}$, Ti, and $\mathrm{O}$ elements.

Figure 3 shows an EELS mapping image where is possible to appreciate that the image is colored whit different colors, the red zone shows the presence of the Pt element, the green zone shows the presence of the Ti element and finally the blue color shows the presence of the Si element due the sample was less of 0.002 mean free path of thickness and flat was possible to identify the exactly position of the different elements on the sample. This analysis compares and corroborates the results obtained previously by HAADF and HAADF+EDS indicating that the FIB equipment is a powerful technique to prepare samples to analyze by HAADF and HAADF+EDS and EELS analytical techniques.

\section{References}

[1] H.Konig, et al., J.apply. Phys. 38 (1999) 6142.

[2] J.P. Reithmaier et al., Microelectr. Eng. 23 (1994) 


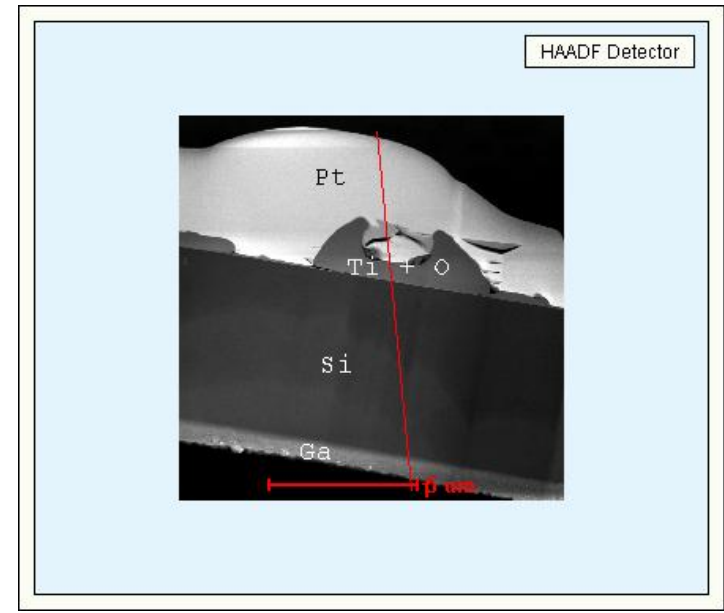

Figure 1. HAADF image showing the contrast and position of the $\mathrm{Pt}, \mathrm{Ti}, \mathrm{O}$ and $\mathrm{Si}$ elements

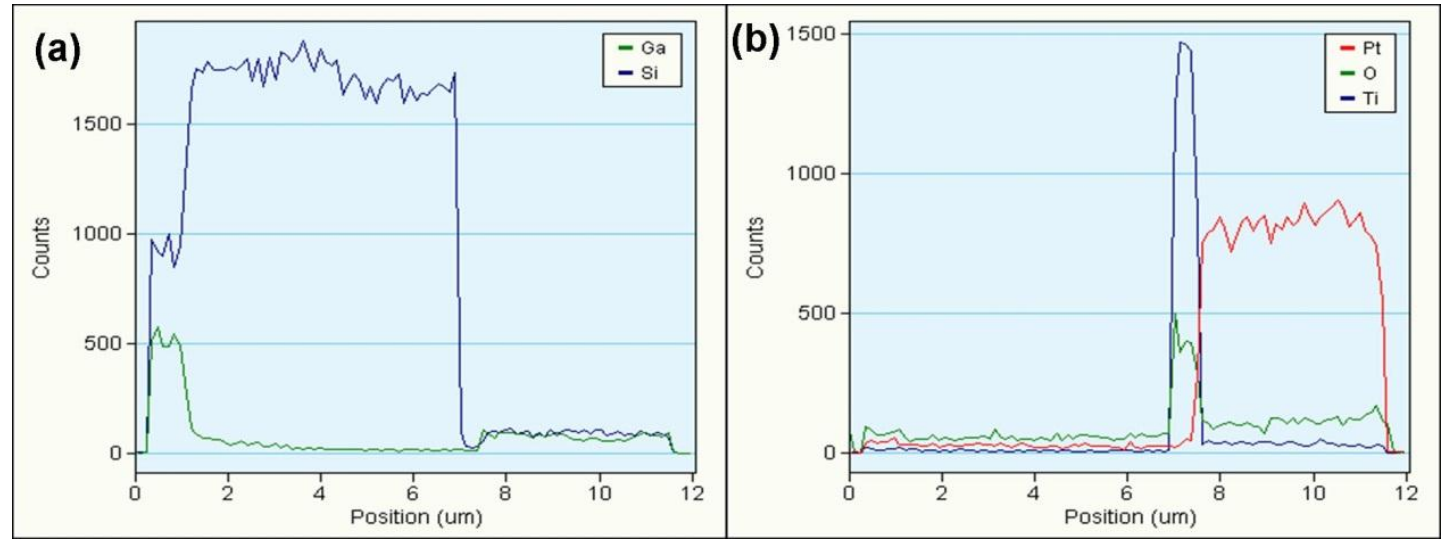

Figure 2. HAADF+EDS images. (a) Line scan of the Ga and Si elements. (b) Line scan of the Pt, $\mathrm{Ti}$ and $\mathrm{O}$ elements.

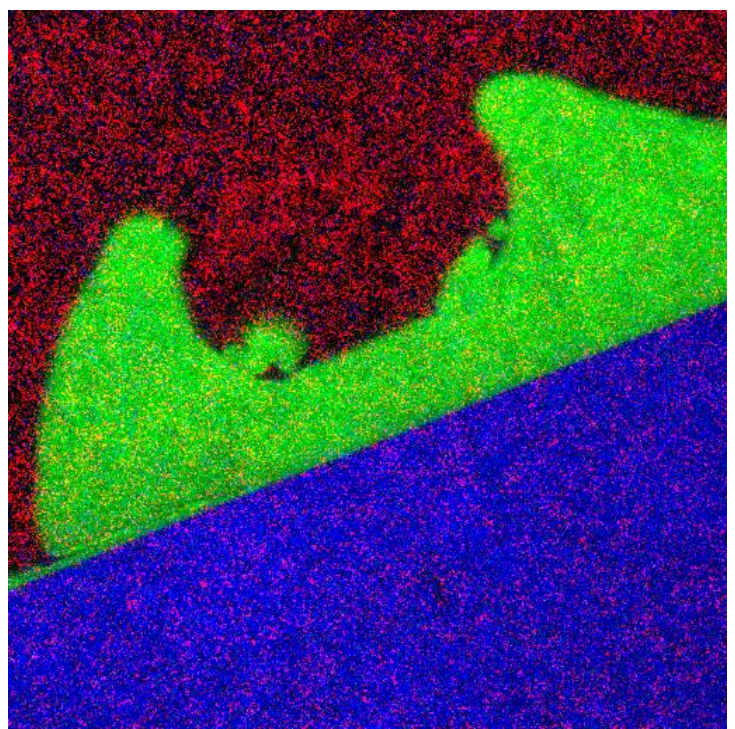

Figure 3. EELS mapping image. Pt (Red color), Ti (Green color) and Si (Blue color). 